\title{
Leukocyte modifications during the first month after foaling in mares and their newborn foals
}

\author{
G. Piccione, M. Rizzo, F. Arfuso, C. Giannetto, S. Di Pietro, M. Bazzano, \\ M. Quartuccio
}

Department of Veterinary Sciences, University of Messina, Italy

\begin{abstract}
During early post-partum period both neonatal foals and peripartum mares are most susceptible to diseases. The aim of this study was to establish physiologic modifications of leukogram during the first month after foaling in mares and their newborn foals. To this end blood samples were collected from nine mares and nine foals (T0-T10), every three days from the 1st day until the 30th day after foaling. Samples were analysed for white blood cell (WBC) count and differential leucocyte counts.

Two-way repeated measure analysis of variance (ANOVA) showed, in postpartum mares WBC showed significant higher values at T0 $(9.02 \pm 0.76)$ in respect to other time points, and at T2 $(8.08 \pm 0.53)$ and $\mathrm{T} 3(7.92 \pm 0.59)$ compared to $\mathrm{T} 1(6.98 \pm 0.43)$, whereas in foals lower WBC values at T0 (6.11 \pm 0.49$)$ compared to other experimental periods except $\mathrm{T} 1(6.90 \pm 0.94)$, and at $\mathrm{T} 1$ compared to $\mathrm{T} 8$ (7.95 \pm 0.61$)$ and $\mathrm{T} 10(7.90 \pm 0.36)$ were observed.

The differential leucocyte counts showed significant modifications in the percentage of neutrophils $(\mathrm{P}<0.001)$ and lymphocytes $(\mathrm{P}<0.001)$ both in postpartum mares and in foals during the experimental period.

Furthermore ANOVA showed significant differences between postpartum mares and foals $(\mathrm{P}<0.01)$ in all studied parameters, and between postpartum mares and control mares in WBC and neutrophils values.

The obtained results provide suitable information about the influence of foaling on leukogram of periparturient mares and reveal WBC dynamics in newborn foals during the first month post-partum.
\end{abstract}

Key words: Foals, Growing, Haematological parameters, Leukocytes, Mares

\section{Introduction}

The early post-natal period is characterized by an increased susceptibility to infectious diseases both in neonatal foals and peripartum mares (Dolente 2004, Knottenbelt et al. 2004). The neonatal period is considered a transitional phase from fetal to extrauterine life. At this time, physiological and metabolic organs and systems have to meet the new challenges of extrauterine environment (Rossdale 2004). At birth the oral nutrient intake becomes the sole source of nutrition; the colostrum's intake shortly after birth is critical for newborn survival as it ensures the transfer of passive immunity (Knottenbelt et al. 2004). Foals live in an environment heavily populated by bacteria, many of which are capable of causing disease

Correspondence to: G. Piccione, e-mail: giuseppe.piccione@unime.it, tel.: +39090 3503584 
Table 1. Mean values \pm SD of physiological, haematological and biochemical parameters obtained from newborn foals.

\begin{tabular}{|c|c|c|c|c|}
\hline \multicolumn{5}{|c|}{ Physiological Parameters } \\
\hline Body temperature & \multirow{2}{*}{\multicolumn{2}{|c|}{$\begin{array}{c}\text { Heart Rate (beats/min) } \\
115 \pm 6\end{array}$}} & \multirow{2}{*}{\multicolumn{2}{|c|}{$\begin{array}{c}\text { Respiratory Rate (breath/min) } \\
36 \pm 4\end{array}$}} \\
\hline $38 \pm 0.5$ & & & & \\
\hline \multicolumn{5}{|c|}{ Haematological Parameters } \\
\hline$R B C\left(10^{6} / \mu L\right)$ & $H b(g / d L)$ & $H c t(\%)$ & Plt $\left(10^{3} / \mu L\right)$ & $W B C\left(10^{3} / \mu L\right)$ \\
\hline $9.58 \pm 0.49$ & $14.71 \pm 1.00$ & $39.90 \pm 2.80$ & $41.61 \pm 1.17$ & $6.11 \pm 0.49$ \\
\hline \multicolumn{5}{|c|}{ Biochemical Parameters } \\
\hline Total Proteins $(g / d L)$ & Albumin $(g / d L)$ & Globulins $(g / d L)$ & Urea $(\mathrm{mmol} / \mathrm{L})$ & Creatinine $(\mu \mathrm{mol} / \mathrm{L})$ \\
\hline $4.62 \pm 0.17$ & $2.59 \pm 0.09$ & $2.21 \pm 0.10$ & $3.7 \pm 0.2$ & $200 \pm 42$ \\
\hline Total Bilirubin $(\mu \mathrm{mol} / L)$ & Triglycerides (mg/dL) & AST (U/L) & $\operatorname{ALT}(\mathrm{U} / \mathrm{L})$ & Glucose (mg/dL) \\
\hline $28.5 \pm 10$ & $101.81 \pm 8.30$ & $14 \pm 6$ & $221 \pm 30$ & $148 \pm 10$ \\
\hline
\end{tabular}

(Giguère and Polkes 2005). Microbial infections constitute a large threat to health in young foals and have been estimated to account for $33 \%$ of foal deaths during the first two months of life (Platt 1973, Haas et al. 1996). The haematological profile represents a useful screening and diagnostic test that can promptly provide valuable information about the health status (Bazzano et al. 2014). In horses, age-related changes in hematological parameters preclude the use of adult reference values in the evaluation of foals. The objective of this study was to evaluate the leukocyte count modifications during the first month after foaling in mares and their newborn foals.

\section{Materials and Methods}

Nine mares (7-9 years) with their respective newborn foals ( 5 females and 4 males) and 7 healthy non-pregnant non-lactating mares (control group, 6-10 years) of different breeds (Italian Saddle, Anglo-Arabian) were enrolled in the study with the informed owner consent. All foals were born full term between February and April 2014. The mares' mean gestation length was $340 \pm 10$ days. Animals were housed in individual straw-bedded boxes $(4.0 \times 3.5 \mathrm{~m})$ at the same breeding centre and were kept under natural environmental conditions. At birth and during the experimental period all foals underwent a clinical examination (evaluation of body temperature, heart rate, respiratory rate, auscultation of lungs and heart, external palpation of the umbilicus), moreover routine haematology and plasma biochemistry testing were assessed at the beginning of the study (Table 1). Mares were subjected to daily clinical examination over the first three days after foaling (evaluation of body temperature, heart rate, respiratory rate, external genitalia, vagina, cervix, uterus and ovaries). During post-partum, transrectal ultrasound exams were weekly performed to ensure the normal involution of the uterus using the M-Turbo ${ }^{\circledR}$ ultrasound system (SonoSite, London, United Kingdom).

Both postpartum mares and control mares were fed twice a day $(7.30 \mathrm{am} ; 5.00 \mathrm{pm})$ and water was available ad libitum. Diet consisted of hay (first cut meadow hay, sun cured, late cut; $6.9 \%$ crude protein on average) and concentrates (crude protein 16\%, crude fat $6 \%$, crude fibre $7.35 \%$, ash $10.09 \%$, sodium $0.46 \%$, lysine $0.85 \%$, methionine $0.35 \%$, omega-3 $0.65 \%$ ) differed for two mares groups in quantity: postpartum mares received $6 \pm 1 \mathrm{~kg} /$ day hay and $5 \pm 0.5$ $\mathrm{kg} /$ day concentrates while control mares received $5 \pm 0.5 \mathrm{~kg} /$ day hay and $2 \pm 0.5 \mathrm{~kg} /$ day concentrates. Foals had access to mares' breast milk ad libitum.

All treatments, housing and animal care were carried out in accordance with the standards recommended by the EU Directive 2010/63/EU for animal experiments.

Sampling was performed in the morning $(08.00$ am) at 11 time points (T0-T10), every three days from the $1^{\text {st }}\left(12-24 \mathrm{~h}\right.$ from foaling) until the $30^{\text {th }}$ day after foaling. Blood was collected by jugular venipuncture into vacuum tubes containing ethylenediaminetetraacetic acid (EDTA) (Terumo Co., Tokyo, Japan). All blood samples were refrigerated and analysed for complete blood count within $6 \mathrm{~h}$ from the collection. All samples were tested for complete blood counts and leukocyte differentiation. The number of total leukocytes was measured using an automated haematology analyzer (HeCo Vet C, SEAC, Florence, Italy). For leukocyte identification and counting, a manual analysis was performed on all samples. Two peripheral blood smears were performed for each sample. After air-drying, the slides were stained through „Dif-Stain” kit (Titolchimica srl, Rome, Italy). The same laboratory professional has later performed the microscopic analysis of blood films by using an optical microscope (Nikon Eclipse e200, Nikon Instruments Europe BV, Amsterdam, Netherlands). A manual 200-cell differential count was performed on each 

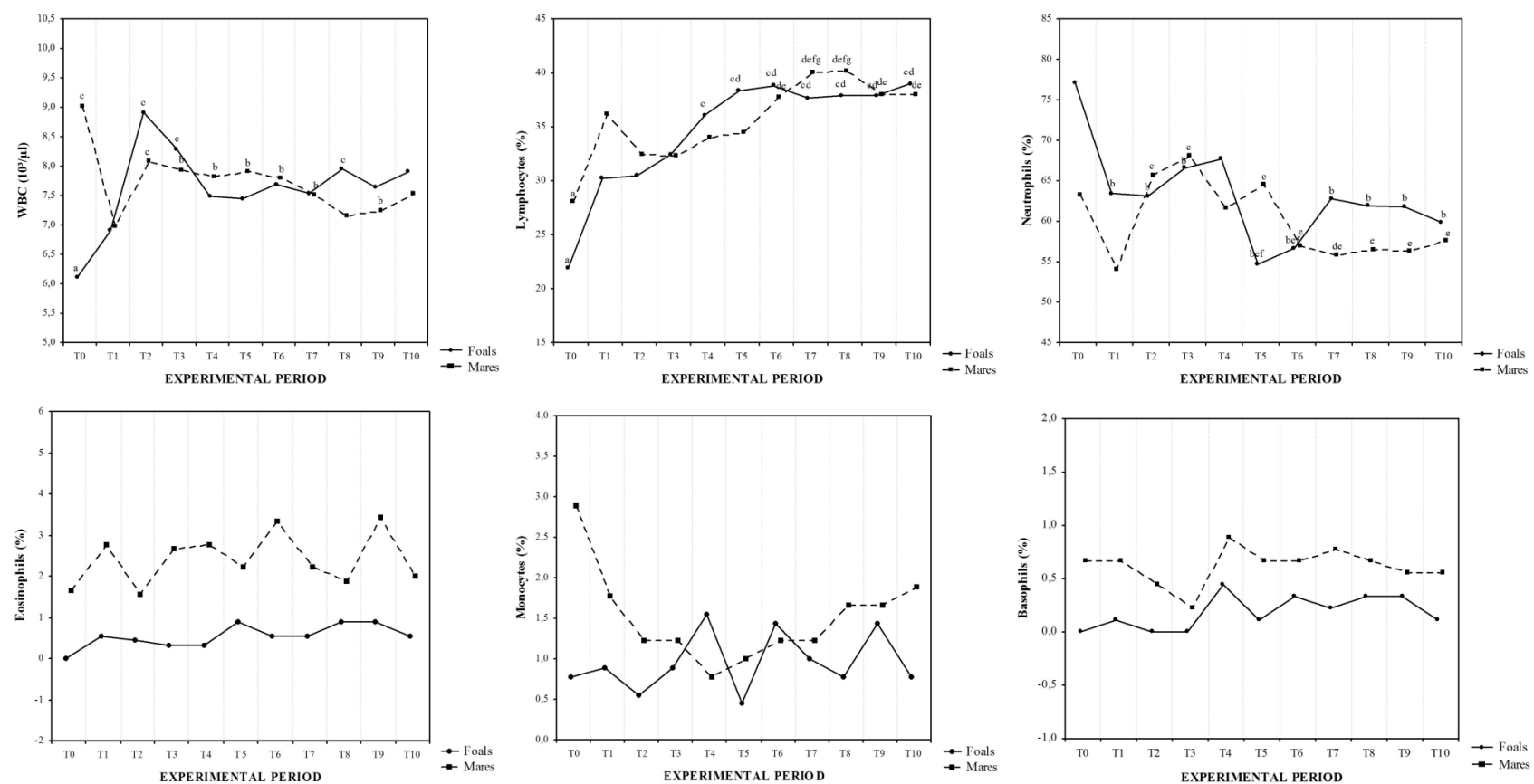

Fig. 1. The trend of mean values together with the relative statistical significances of leukocyte parameters obtained every three days during the first month post-partum (T0-T10). Significances (TIME): a vs all, b vs T0, c vs T1, d vs T2, e vs T3, f vs T4, g vs T5.

blood film. For each animal, the leukocyte differential count was calculated by averaging of the data recorded from each blood film of the same sample.

Two-way analysis of variance (ANOVA) for repeated measures was applied to evaluate changes in the immunologic parameters over time (within-subjects), differences between foals and postpartum mares, and between postpartum mares and control mares. $P$ value $<0.05$ was considered statistically significant. Data were analyzed using statistical software STATISTICA 7 (StatSoft Inc., Tulsa, OK, USA).

\section{Results}

Two-way ANOVA showed a statistically significant effect of time $(P<0.001)$ on WBC count both in postpartum mares and foals (Figure 1). No significant effect of time was observed in control mares.

In particular in postpartum mares WBC showed significant higher values at $\mathrm{T} 0(9.02 \pm 076)$ respect to other time point, and at $\mathrm{T} 2(8.08 \pm 0.53)$ and $\mathrm{T} 3$ $(7.92 \pm 0.59)$. compared to $\mathrm{T} 1(6.98 \pm 0.43)$. In foals Bonferroni's post hoc test showed significantly lower WBC values at T0 $(6.11 \pm 0.49)$ compared to other experimental periods except $\mathrm{T} 1(6.90 \pm 0.94)$, and at $\mathrm{T} 1$ compared to T8 $(7.95 \pm 0.61)$ and $\mathrm{T} 10(7.90 \pm 0.36)$.

The differential leucocyte counts showed significant modifications in the percentage of neutrophils $(\mathrm{P}<0.001)$ and lymphocytes $(\mathrm{P}<0.001)$ both in postpar- tum mares and in foals during the experimental period (Figure 1). Lymphocytes count (\%) in postpartum mares was lower at T0 $(28.11 \pm 1.45)$ in respect to all time points, at T2 (32.44 \pm 2.55$)$ and T3 $(32.33 \pm 2.87)$ compared to T6-T10, and at T4 $(34.00 \pm 2.91)$ and T5 (34.44 \pm 2.35$)$ compared to $\mathrm{T} 7(40.00 \pm 2.50)$ and $\mathrm{T} 8$ (40.11 \pm 2.89$)$; in foals lymphocytes showed lower values at T0 $(21.88 \pm 2.02)$ compared to other time point, at T1 $(30.22 \pm 2.33)$ and $\mathrm{T} 2(30.44 \pm 2.69) \mathrm{com}-$ pared to T4-T10 and at T3 (32.44 \pm 2.50$)$ compared to T6-T10. Neutrophils (\%), in postpartum mares, showed significantly increased values at $\mathrm{T} 2$ $(65.66 \pm 10.82)$ as compared to $\mathrm{T} 1(63.22 \pm 8.01)$ and $\mathrm{T} 7$ $(55.77 \pm 6.15)$, at $\mathrm{T} 3(68.00 \pm 3.80)$ as compared to $\mathrm{T} 1$, T6-T10; at T5 (64.44 \pm 1.42$)$ as compared to T1; foal's neutrophils assumed higher values at T0 $(77.11 \pm 3.51)$ in respect to other time point and decreased at T5 (54.66 \pm 3.93$)$ and $\mathrm{T} 6(56.66 \pm 5.56)$ in respect to $\mathrm{T} 3$ (66.56 \pm 7.56$)$ and T4 (67.66 \pm 4.60$)$.

Furthermore, ANOVA showed significant differences between foals and postpartum mares $(\mathrm{P}<0.01)$ in all studied parameters (Figure 2) and between postpartum mares and control mares in WBC and neutrophils values (Figure 3).

\section{Discussion}

The present study shows how leukogram changes in postpartum mares and their newborn foals, but not 

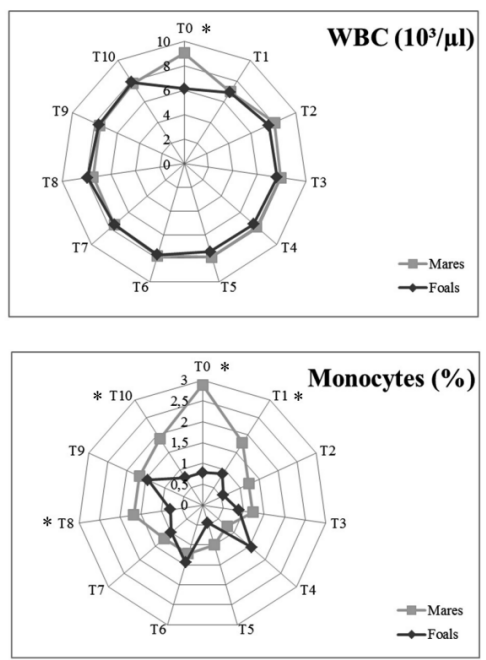
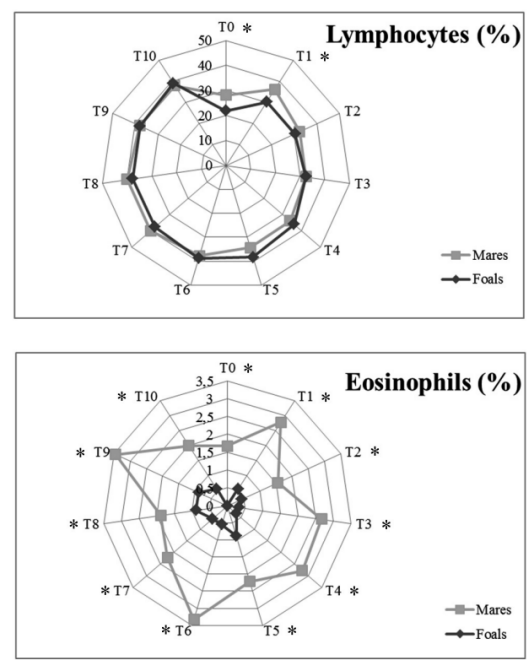
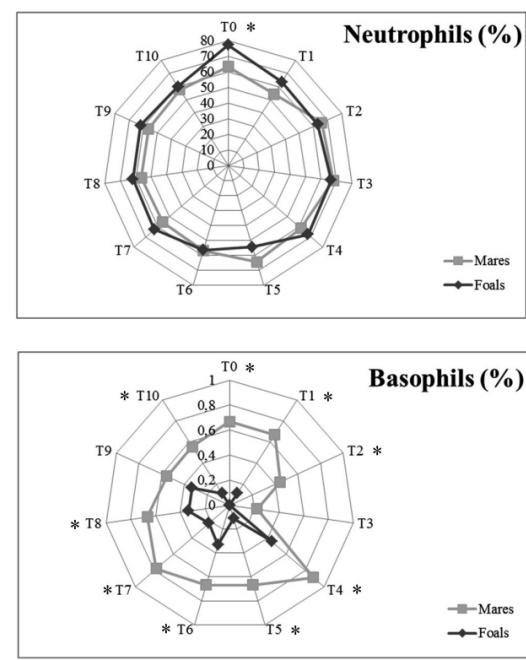

Fig. 2. Radar graph representing the mean values of leukocyte parameters in mares $(\longrightarrow \bullet)$ and foals ( $\longrightarrow$-). Each axis displays the proportion of each leukocyte subset within a given time point (T0-T10). Significances (GROUP): * vs Foals.
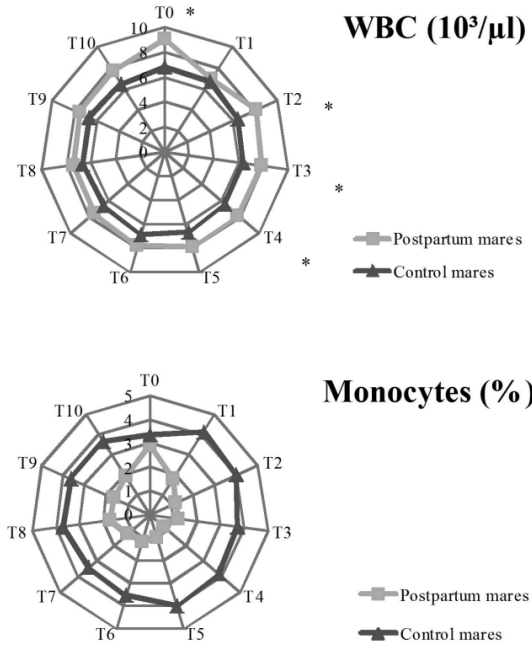

Monocytes (\%)

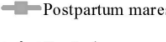

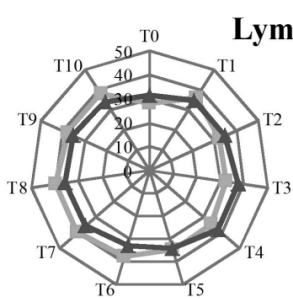

ymphocytes $(\%)$
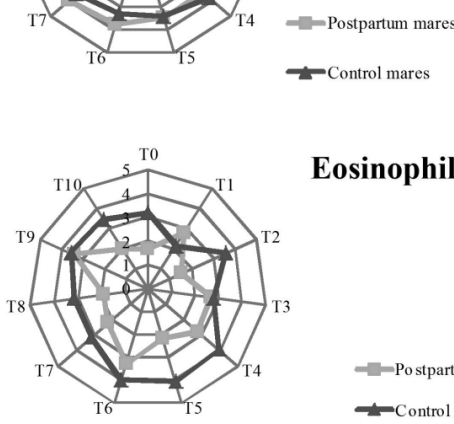

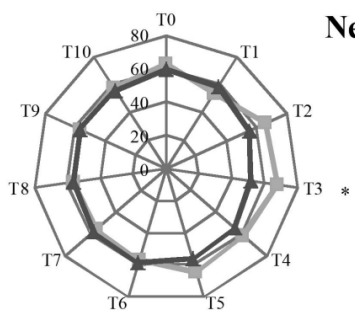

Neutrophils $(\%)$

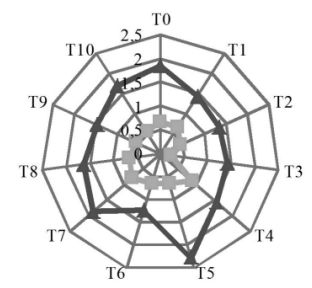

Basophils (\%)
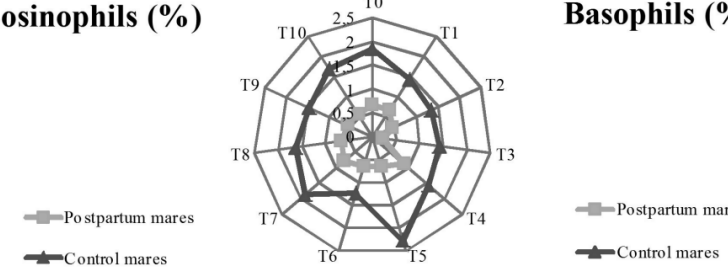

$\simeq$ Control mares

Fig. 3. Radar graph representing the mean values of leukocyte parameters in postpartum mares ( $\longrightarrow$-) and control mares ( $\longrightarrow-$ ). Each axis displays the proportion of each leukocyte subset within a given time point (T0-T10). Significances (GROUP): * vs control mares.

in control mares. According to previous studies on periparturient mares (Harvey et al. 1994, Aoki et al. 2013, Bazzano et al. 2014, Mariella et al. 2014), our results showed a significant increase in WBC within $24 \mathrm{~h}$ after foaling. The peak in WBC could be due to physical stress associated with delivery (Aoki et al. 2013) and consequent release of cortisol and catecholamine (Davies Morel 1993). A significant rise in blood cortisol concentrations was observed in pregnant mares in the imminence of parturition as well (Nagel et al. 2012).

It is known that these stress hormones induce significant changes in absolute numbers and relative proportions of leukocytes in the blood. In fact, changes in blood leukocyte numbers were used as an indirect measure for changes in plasma cortisol before methods were available to directly assay the hormone (Hoagland et al. 1946), and numerous studies in humans have shown that glucocorticoid hormones induce significant changes in blood leukocyte distribution (Dhabhar et al. 1996). Studies have also delineated the rapid and significant effects of catecholamine hormones in mediating stress-induced changes in blood leukocyte distribution (Redwine et al. 2003).

At birth foals showed lower average values in total 
leukocyte count. Between $\mathrm{T} 3$ and $\mathrm{T} 10$ a reduction in WBC was found. These variations are considered physiological and have been previously described in foals of other breeds (Harvey et al. 1994, Stoneham 2006, Aoki and Ishii 2012).

A significant increase in neutrophils was observed in the first $24 \mathrm{~h}$ of the foal's life. This increase is probably due to the large increase in circulating neutrophils in response to the peak level of cortisol in the fetal circulation that occurs in this phase (Stoneham 2006, Axon and Palmer 2008). This occurs only in healthy foals and it is directly related to endogenous steroid production, maturity of the adrenocortical system and consequently the foal's maturity at birth (Rossdale 2004). As previously found in newborn foals (Waelchli et al. 1994, Flaminio et al. 1999, Smith III et al. 2002, Aoki and Ishii 2012, Aoki et al. 2013), in this study a progressive increase in lymphocytes during the first month of life was observed. At birth, healthy foals have approximately 2.5 times more neutrophils than lymphocytes, variable number of monocytes, and no eosinophils (Welles 2010). Effectively, eosinophils are low during the first month of life, and slowly increase in number, probably in response to intestinal parasite exposure (Welles 2010). As reported by other researches (Harvey 1990, Curcio and Nogueira 2012) our results showed a low number of monocytes and basophils during the neonatal period.

In conclusion, obtained results provide suitable information about the influence of foaling on leukogram of periparturient mares and reveal WBC dynamics in newborn foals during the first month post-partum supporting clinicians to better interprete clinical data and diagnose equine neonatal diseases.

\section{References}

Aoki T, Honda H, Ishii M (2013) Immunologic profiles of peripheral blood leukocytes and serum immunoglobulin $\mathrm{G}$ concentrations in perinatal mares and neonatal foals (heavy draft horse). J Equine Vet Sci 33: 989-995.

Aoki T, Ishii M (2012) Hematological and Biochemical Profiles in Peripartum Mares and Neonatal Foals (Heavy Draft Horse). J Equine Vet Sci 32: 170-176.

Axon JE, Palmer JE (2008) Clinical Pathology of the Foal. Vet Clin North Am Equine Pract. 24: 357-385.

Bazzano M, Giannetto C, Fazio F, Rizzo M, Giudice E, Piccione G (2014) Physiological adjustments of haematological profile during the last trimester of pregnancy and the early post-partum period in mares. Anim Reprod Sci 149: 199-203.

Curcio BR, Nogueira CEW (2012) Newborn adaptations and healthcare throughout the first age of the foal. Anim Reprod 9: 182-187.

Dhabhar FS, Miller AH, McEwen BS, Spencer RL (1996) Stress-induced changes in blood leukocyte distribution. Role of adrenal steroid hormones. J Immunol 157: 1638-1644 .
Davies Morel MCG (1993) Equine reproductive physiology, breeding and stud management. Farming Press Books, Ipswich.

Dolente BA (2004) Critical peripartum disease in the mare. Vet Clin North Am Equine Pract 20: 151-165.

Flaminio MJ, Rush BR, Shuman W (1999) Peripheral blood lymphocyte subpopulations and immunoglobulin concentrations in healthy foals and foals with Rhodococcus equi pneumonia. J Vet Intern Med 13: 206-212.

Giguère S, Polkes AC (2005) Immunologic Disorders in Neonatal Foals. Vet Clin North Am Equine Pract 21: 241-272.

Haas SD, Bristol F, Card CE (1996) Risk factors associated with the incidence of foal mortality in an extensively managed mare herd. Can Vet J 37: 91-95.

Harvey JW, Asquith RL, McNulty PK, Kivipelto J, Bauer JE (1984) Haematology of foals up to one year old. Equine Vet J 16: 347-353.

Harvey JW, Asquith RL, Pate MG, Kivipelto J, Chen CL, Ott EA (1994) Haematological findings in pregnant, postparturient and nursing mares. Comp Haematol Int 4: 25-29.

Harvey JW (1990) Normal hematologic values. In: Koterba AM, Drummond WH, Kosch PC (Eds) Equine clinical neonatology. Lea \& Febiger, Philadelphia, pp 561-570.

Hoagland H, Elmadjian F, Pincus G (1946) Stressful psychomotor performance and adrenal cortical function as indicated by the lymphocyte response. J Clin Endocrinol Metab 6: 301-311.

Knottenbelt DC, Holdstock N, Madigan JE (2004) Equine neonatology Medicine and surgery. Saunders Ltd., Edinburg, London, New York, Oxford, Philadelphia, St Louis, Sydney, Toronto.

Mariella J, Pirrone A, Gentilini F, Castagnetti C (2014) Hematological and biochemical profiles in Standardbred mares during peripartum. Theriogenology 81: 526-534.

Nagel C, Erber R, Bergmaier C, Wulf M, Aurich J, Mostl E, Aurich C (2012) Cortisol and progestin release, heart rate and heart rate variability in the pregnant and postpartum mare, fetus and newborn foal. Theriogenology 78: 759-767.

Platt $\mathrm{H}$ (1973) Etiological aspects of perinatal mortality in the Thoroughbred. Equine Vet J 5:116-120.

Redwine L, Snow S, Mills P, Irwin M (2003) Acute psychological stress: effects on chemotaxis and cellular adhesion molecule expression. Psychosom Med 65: 598-603.

Rossdale PD (2004) The maladjusted foal: influence of intrauterine growth retardation and birth trauma. In: Proceedings of the 50th Annual Convention of the American Association of Equine Practitioners. Denver AAEP, Denver, CO. pp 75-126.

Smith R 3rd, Chaffin MK, Cohen ND, Martens RJ (2002) Age-related changes in lymphocyte subsets of quarter horse foals. Am J Vet Res 63: 531-537.

Stoneham SJ (2006) Assessing the newborn foal. In: Paradis MR (Eds) Equine Neonatal Medicine. Philadelphia pp 7-10.

Waelchli RO, Lutz H, Hermann M, Ruegg C, Eggenberger E (1994) Hematological reference values for foals in the first two months of life. Schweiz Arch Tierheilkd 136: $127-136$.

Welles EG (2010) Interpretation of equine Leukocyte Responses. In: Weiss DJ, Wardrop KJ (Eds) Schalm's Veterinary Hematology, 6th ed., Blackwell Publishing Ltd., Oxford, pp 314-320. 